\title{
L'etruscità di Persio e la sua posizione politica
}

\author{
ILARIA RAMELLI
}

\section{RIASSUNTO SUMMARY}

L'articolo propone un'analisi degli elementi etruschi nell'opera e nella cultura del poeta romano-etrusco

Persio di Volterra, e una presentazione critica del pensiero politico di questo autore, nel suo contesto filosofico, storico e religioso.
The article proposes an analysis of the Etruscan elements in the work and in the culture of the Roman-Etruscan poet Persius from Volterra and a critical presentation of the politic thought of this author, in his philosophical, religious and historical context.

L'etruscità di Persio, il poeta stoico nato a Volterra nel 34 d.C., messa in luce già almeno parzialmente da Henri Bardon e da Antonio La Penna ${ }^{1}$, traspare in primo luogo dal suo stesso nome, Aules, che appare una forma ibrida tra l'etrusco Aule e la sua forma latinizzata Aulus. Persio rimase a Volterra, dove visse insieme con la madre Fulvia Sisennia e ricevette i primi gradi della sua istruzione, fino a dodici anni, come ci informa la Vita Persi ${ }^{2}$ al cap. 4: Studuit Flaccus usque ad

\footnotetext{
1 H. Bardon, Perse, ou l'homme du refus, "Rev. Belge de Phil. et d'Hist." 53 (1975), pp. 24 sgg., part. 40-43; A. LA PENnA. Persio e le vie nuove della satira latina, intr. a PERSIO, Satire, Milano $1988,3 .^{a}$ ed., p. 24 ; io stessa ho in preparazione un libro sulla cuitura etrusca alla fine dell'indipendenza in cui dedico un capitolo agli Stoici romano-etruschi di età neroniana, con bibliografía.

2 La vita Auli Persi Flacci è attribuita nei codici al grammatico Valerio Probo che commentò anche le Satire di Persio. Oggi sono state proposte anche altre paternità, come quella svetoniana. Sembra comunque che il nucleo d questa vita sia antico e attendibile; cfr. E. Marmorale, Persio, Firenze 1956, 2. ${ }^{a}$ ed., pp. 109 ss.; E. Paratore, Biografia e poetica di Persio, Firenze 1968, p. 1 ss.
} 
annum XII aetatis suae Volaterris, inde Romae. la sua conoscenza dei costumi etruschi si rivela con evidenza nelle sue Satire, laddove, riferendo esempi e situazioni di vita quotidiana, sceglie proprio personaggi e usi inequivocabilmente etruschi ${ }^{3}$.

Per esempio, già nella satira I Persio ricorda la magistratura di edile nella città di Arezzo, naturalmente etrusca, e critica l'Etrusco che, fiero di una magistratura italica, in quanto edile ha spezzato in Arezzo le misure false: Persio dichiara di non volere come lettore uno sese aliquem credens, Italo quod honore supinus, / fregerit heminas Arreti aedilis iniquas (Pers. I 129-130). II poeta, dunque, qui deride i suoi conterranei che si vantavano anche di una carica pubblica di poco conto in una città etrusca ${ }^{4}$.

In un altro punto Persio critica la tendenza dei suoi conterranei a vantarsi della propria nobiltà: è quando dice che gli Etruschi nobili andavano fieri delle loro genealogie che potevano essere tracciate addietro fino alla millesima generazione. $\dot{E}$ stato osservato ${ }^{5}$ come questo risalire addietro il più possibile nel tempo, per le famiglie etrusche, avesse un significato particolare in rapporto all'autoctonia del popolo etrusco, proclamata dagli Etruschi stessi, come appare evidente dal l'oracolo della Ninfa Vegoia, che presuppone gli Etruschi stanziati nella terra Etruria fin dall'origine del mondo ${ }^{6}$. Questo tema è affrontato nella satira III, dove Persio, rivolgendosi a un "giovin signore" da istruire, mette a fuoco il valore della vera nobiltà per affermare che essa non si identifica con la nobiltà della stirpe,

\footnotetext{
3 J. HeUrgon, Les eléments etrusques dans les Satyres de Perse, "Revue des Études Latines" 44 (1966), pp. 30-32; M. SoRDI, Lo Stoicismo in Etruria nel I secolo a.C., in Die Integration der Etrusker und das Weiterwirken etruskischen Kulturgutes im republikanischen und kaiserzeitlichen Rom, hrsg. von L. Aigner Foresti, Wien 1998 (Sitzungsberichte / Österreichische Akademie der Wissenschaften, Philosophisch-historische Klasse, 658), pp. 337-44, part. p. 339.

4 Sulla vita pubblica degli Etruschi nel I secolo nelle città italiche cfr. Valvo, Onomastica e integrazione, in Die Integration der Etrusker, cit., pp. 187-203.

5 Cfr. M. TORELLI, Senatori etruschi della tarda repubblica e dellimpero, "Dialoghi di Archeologia" 3 (1969), pp. 285-363, part. 341: Le famiglie etrusche compivano "uno sforzo deliberato per ricercare origini antiche» e questo era per loro "un'ossessione"; cfr. S. MONTERO, Política y adivinación en el Bajo Imperio, Bruxelles 1991, p. 35.

6 Cfr. R. Turcan, Encore la prophétie de Végoia, in Mélanges Heurgon, 11, Rome 1976 , pp. 1009-1019; A. VALVO, La profezia di Vegoia. Proprietà fondiaria e aruspicina in Etruria nel I sec. a. C., Roma 1988: F. H. Massa-Pairault, Lasa Vecu-Lasa Vecuvia, "Dialoghi di Archeologia" VI 3, Roma 1988, pp. 133-143; Eiusd. Regards sur Volsinii Variations sur le theme de la survivance di la culture étrusque, in Die Integration der Etrusker, cit, pp. 421-433, part. 424-425.
} 
bensì con quella morale: An deceat pulmonem rumpere ventis / stemmate quod Tusco ramum millesime ducis? (Pers. III 37-38). II cavaliere etrusco Persio aveva avuto occasione di conoscere i nobili etruschi fierissimi delle loro origini avite. Egli proclama invece con altrettanta fierezza di non vantare una discendenza di molte generazioni e di non saper risalire oltre alla quinta o alla sesta generazione dei suoi antenati: quaere ex me, quis mihi quartus / sit pater: haud prompte, dicam tamen; adde etiam unum, / unum etiam: terrae est filius ${ }^{7}$ (Pers. VI 57 sgg.).

Un altro elemento che, forse, potrebbe rivelare un indizio di etruscità di Persio è lo stile aspro, involuto, sintetico e difficile che presenta indubbie somiglianze con quello della lettera di Cecina a Cicerone ${ }^{8}$. Purtoppo non è possibile trovare paralleli analoghi né con l'etrusco Musonio né con Trasea Peto, poiché per entrambi non ci sono pervenute opere (come è noto, le diatribe di Musonio furono composte dal suo discepolo Arriano).

Anche quando, nella sua satira II, Persio deve cercare esempi di superstizione da criticare, li trova presso i suoi conterranei, ligi ovviamente all'aruspicina ${ }^{9}$ : ignovisse putas [sc. lovem], quia, cum tonat, ocius ilex / sulphure discutitur sacro quam tuque domusque? / An quia non fibris ovium Ergennaque iubente / triste iaces lucis evitandumque bidental, / idcirco stolidam praebet tibi vellere barbam / luppiter? Aut quidnam est qua tu mercede deorum / emeris auriculas? Pulmone et lactibus unctis? (Pers. II 24-30). II riferimento all'ars fulguratoria e alle viscere dell'extispicina, e soprattutto il nome etrusco di Ergenna, che designa chiaramente un aruspice, non lasciano dubbi sul contesto etrusco della scenetta. Non sembra di dovere intendere una critica di Persio alla Etrusca disciplina e alla religiosità etrusca in sé. Anzi, Persio è uno spirito profondamente religioso e, come ho avuto occasione di analizzare più estesamente in altra sede ${ }^{\circ}$, la sua caratterizzazione di Giove come suprema divinità, custode della vita morale degli uomini, sembra rivelare un profondo influsso del Tinia etrusco. Que-

7 L'espressione "figlio della terra" equivale alla nostra "figlio di nessuno", e indica una persona i cui genitori sono ignoti, ma può richiamare anche un'idea di autoctnonia.

8 Su A. Caecina cfr. CAPDEVILLE, Sources, pp. 13-21; 23-25; Eiusd. I Caecina e Volterra, in Aspetti della cultura di Volterra Etrusca fra l'Età del Ferro e l'Età Ellenistica. Atti del XIX Convegno di Studi Etruschi e Italici, Volterra, 15-19 ottobre 1995; Eiusd. Die Rezeption, pp. 400-401 per il rapporto politico ed epistolare con Cicerone.

9 Sugli sviluppi dell'Etrusca Disciplina nel I secolo si veda con bibliografía il mio cit. La cultura etrusca alla fine dellindependenza, cap. II $\$ 1$.

10 La concezione di Giove negli Stoici romani di età neroniana, "Rend. Ist. Lomb., Classe di Lettere", 131 (1997), pp. 293-320. 
Ili che Persio critica sono piuttosto i superstiziosi che credono, formalisticamente, di guadagnarsi il favore degli dèi con i meri sacrifici, mentre Persio, alla fine della satira II, precisa che quello che Giove desidera è piuttosto l'impegno morale nel perseguimento della virtù (Pers. II 71-75) ${ }^{11}$.

Sempre verso la fine della satira II, infatti, il riferimento alla sobrietà del culto etrusco delle origini riveste una valenza certamente positiva e costituisce anzi un modello, secondo Persio, del corretto modo di adorare gli dèi: egli infatti biasima il lusso dei templi, analogamente a quanto fa Seneca, che tuttavia biasima ogni forma di culto materiale in quanto irrispettosa dell'autarchia della divinità (Ep. 95, 37 e 72; 110, 18-20; $119,7)$; Persio invece critica solo il culto lussuoso, che contempla suppellettili preziose, e ricorda con rimpianto la sobrietà dei tempi in cui nei santuari non c'era ancora l'oro: aurum vasa Numae Saturniaque inpulit aera / Vestalisque urnas et Tuscum fictile mutat. / o curvae in terris animae et caelestium inanes, lquid iuvat hoc, templis nostros inmittere mores / et bona dis ex hac scelerata ducere pulpa? ${ }^{12} /$... peccat et haec, peccat, vitio tamen utitur: at vos / dicite, pontifices, in sancto quid facit aurum? (Pers. II 59-69). I vasi etruschi di terracotta sembrano identificabili con i buccheri e in generale con gli oggetti fittili che l'artigianato etrusco produceva in abbondanza. Che, del resto, Persio fosse familiare a questi oggetti e alla loro produzione è rivelato da un passo iniziale della satira III, in cui egli paragona un giovane da istruire a terracotta da modellare e mostra di distinguere i suoni che emettono gli oggetti di terracotta quando sono percossi: sonat vitium percussa, maligne / respondet viridi non cocta fidelia limo. / Udum et molle lutum es, nunc nunc properandus et acri / fingendus sine fine rota (Pers. III 21 sgg.).

Un altro elemento "etrusco" che si rileva chiaramente nella vita di Persio appare la costante presenza delle donne della sua famiglia e il suo immenso rispetto per loro ${ }^{13}$ : ho avuto modo di mostrare come questa particolare valutazione dell'elemento femminile sia presente

\footnotetext{
11 Quin damus id superis, de magna quod dare lance / non possit magni Messallae lippa propago: / compositum ius fasque animo, sanctosque recessos / mentis, et incoctum generoso pectus honesto. / Haec cedo ut admoveam templis et farre litabo.

12 Sul concetto di «carne" in Persio e sulle sue connessioni con quello paolino si veda $M$. Sord, Aerumnosi Solones, “Aquileia Nostra» 45-46 (1974-75), pp. 278-279.

${ }_{13}$ Sullimportanza della donna nella società etrusca cfr. M. SoRDl, La donna etrusca, in Misoginia e maschilismo in Grecia e in Roma, Genova 1981, pp. 49-67, oggi in Ead., Prospettive di storia etrusca, Como 1995, pp. 159-173.
} 
anche in Musonio, probabilmente proprio come eredità della sua cultura etrusca ${ }^{14}$. Nella Vita Persi, 6,entro il ritratto morale di Persio, sono ricordate esplicitamente la sua devozione verso la madre e le parenti, segnatamente la sorella e la zia, la sua mitezza, la verecondia e la pudicizia: fuit morum lenissimorum, verecundiae virginalis, famae pulchrae, pietatis erga matrem et sororem et amitam exemplo sufficientis. Nella Vita Persi, 8, poi, si dice che i versi in lode di Arria Maggiore - si noti, della donna e non di suo marito Cecina Peto- furono cancellati dall'opera di Persio non direttamente dal suo maestro ed editore, Anneo Cornuto, quanto dalla madre di Persio, dietro suggerimento di Cornuto ${ }^{15}$. Le modifiche all'opera di Persio, pubblicata postuma, non vennero operate formalmente da Cornuto stesso, bensi dalla madre di Persio, che evidentemente era la depositaria dei suoi scritti: Persio aveva lasciato la sua opera filosofico-poetica alla madre e non al maestro. In prossimità della morte, Persio lasciò anche il suo mandato testamentario alla madre, che per volontà del figlio diede a Cornuto i settecento libri di Crisippo che Persio conservava nella sua biblioteca (Vita, 7: scriptis ad matrem codicillis, Cornuto rogavit ut daret ... libros circa septingenti Chrysippi sive bibliothecam suam omnem). Sempre a proposito della particolare attenzione per le donne dimostrata da Persio, indice di una cultura che non sottovalutava l'elemento femminile nella società, il poeta nella satira II descrive le preghiere, a suo avviso vane, formulate dalla nonna o dalla zia materna sulla culla del bambino, augurandogli di divenire genero di re e di regine e conteso dalle fanciulle - come nota la Sordi, è l'idea del "principe azzurro" al contrario- ${ }^{16}$, e riporta dei rituali magici che egli probabilmente aveva avuto occasione di vedere di persona: Ecce avia aut metuens divum matertera cunis / exemit puerum frontemque atque uda labella / infami digito et lustralibus ante salivis / expiat, urentis oculos inhibere perita; / tunc manibus quatit et spem macram supplice voto / nunc Licini in campos, nunc Crassi mittit in aedis: / Hunc optent

\footnotetext{
14 Cfr. la mia Introduzione a Musonio Rufo. Diatribe, Frammenti, Testimonianze, Milano 2001.

15 Scripserat in pueritiam Flaccus ...paucos in socrum Thraseae, Arriam matrem, versus, quae se ante virum occiderat. Omnia ea auctor fuit Cornutus matri eius ut aboleret. Su questo ruolo di Anneo Cornuto e sul suo rapporto con Persio e con L'opposizione stolca rinvio alla mia Introduzione in L. Anneo Cornuto, Compendio di Teologia greca, a c. di I. RameIli, Milano 2003.

16 SoRd, La donna etrusca, pp. 49-67.
} 
generum rex et regina, puellae / hunc rapiant; quidquid calcaverit, hic rosa fiet (Pers. II $31 \mathrm{sgg}$.). Persio, che evidentemente aveva sotto gli occhi questi esempi, protesta che non dovrebbero essere le nutrici a innalzare voti con richieste di beni apparenti: ast ego nutrici non mando vota, negato, / luppiter, haec illi, quamvis te albata rogarit (ibid. 39-40). Come è noto, il filosofo contemporaneo di Persio, anch'egli stoico e di origini etrusche, Musonio Rufo di Volsinii, a più riprese nelle sue diatribe insiste sulla necessità di educare allo stesso modo le bambine e i bambini e sull'opportunità che anche le donne pratichino la filosofia (diss. III-IV) ${ }^{17}$. Del resto, il forte senso della famiglia che emerge dalla Vita Persi trova precisa corrispondenza nell'attenzione posta da Musonio alla famiglia, alla sua morale e alla sua sacralità, nelle diatribe XIV e XV, che condannano anche l'esposizione dei bambini e l'interruzione di gravidanza, che ricordano che Zeus stesso è il Protettore della famiglia (ónóvios) e che sembrano scritte in polemica con Teopompo ${ }^{18}$.

Anche una piccola nota gastronomica, legata anch'essa alla presunta mollezza degli Etruschi, potrebbe rivelare l'etruscità di Persio: l'uso toscano di mettere il pepe nel prosciutto è ricordato da Persio nella satira III, dove il prosciutto pepato è presentato come il dono di un cliente marsico al suo avvocato difensore: Disce nec invideas quod multa fidelia putet / in locuplete penu, defensis pinguibus Umbris, / et piper et pernae, Marsi monumenta clientis, maenaque quod prima nondum defecerit orca (III 73-76). II pepe nella satira VI è ricordato come segno di mollezza nelle parole di Bestius, il romano tradizionalista: Ita fit: postquam sapere ${ }^{19}$ urbi / cum pipere et palmis venit nostrum hoc maris expers, /faenisecae crasso vitiarunt unguine pultes (VI 38-40). In effetti l'accusa di mollezza era rivolta tradizionalmente agli Etruschi e Persio stesso, nella satira V, vv. 142 sgg., presenta addirittura la prosopopea della Mollezza (Luxuria, che rende il greco $\tau \rho \cup \phi \eta$ "), e dei suoi

\footnotetext{
17 Cfr. La mia Introduzione a Musonio, cit., e le mie annatazioni alle Diatribe Ili, IV e V tradotte nel medesimo volume.

18 M. SoRol, Storiografia e cultura etrusca nell'Impero romano, in Atti del /l Congresso internazionale etrusco (26 maggio - 2 giugno 1985), I, Roma 1989 (Suppl. di Studi Etruschi), pp. 41-51; Ead., Lo Stoicismo in Etruria, in Die Integration der Etrusker, cit, pp. 337-47 ed eventualmente il mio cit. La concezione di Giove, pp. 293-320.

19 Qui appare chiaro il gioco di parole tra sapere inteso come «sapere» e sapere come "avere sapore": dalla Grecia è giunto il sapere, ma la mollezza è legata anche ai prodotti gastronomici, ai quali ovviamente afferisce l'accezione di sapere come "avere sapore".
} 
principî gaudenti ${ }^{20}$. È interessante che un ulteriore cenno all'Etruria, e in particolare al famigerato vino di Veio, si trovi precisamente in questo passo, laddove Mollezza domanda all'aspirante commerciante se vorrebbe andare per mare e bere il disgustoso vino di Veio: tu mare transilias? Tibi, torta cannabe fulto, / cena sit in transtro Veientanumque rubellum / exhalet vapida laesum pice sessilis obba?, «E tu vorresti attraversare il mare? / Vorresti tu, poggiato a una ritorta gòmena, /cenare sul bancone, e che una caraffa / larga esalasse il rosso vin di Veio / rovinato da pece puzzolente?" (V 146-148).

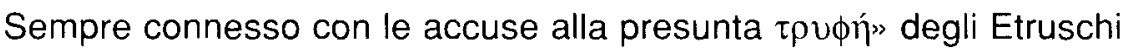
è anche l'accusa di depilarsi, un'accusa che Persio mostra di conoscere e che critica personalmente, adducendo che coloro che rivolgono queste accuse si comportano poi in maniera ancora peggiore (Pers. IV $33 \mathrm{sgg.)}{ }^{21}$. In questo medesimo passo vale forse anche la pena di notare che il parlante biasima uno che si depila il corpo e non si rade però la barba: in una delle sue diatribe finali dedicata espressamente alla questione della barba lo stoico etrusco Musonio consiglia analogamente agli uomini di non radersi la barba (diatriba 21).

\section{NOTE POLITICHE: L'ATTEGGIAMENTO DI PERSIO VERSO IL PRINCIPATO}

Persio, come anche Trasea Peto e Musonio Rufo, stando alle notizie che ci è consentito di ricavare dalle fonti, fece parte della opposizione 'morale' stoica al principato neroniano; $i$ tre erano per altro legati tra loro da vincoli di amicizia: Musonio, come dicevamo, era amico di Trasea Peto, oltre che di Barea Sorano e Rubellio Plauto; anche Persio, come

20 La Moliezza viene introdotta per dissuadere colui che vuole dedicarsi al commercio marittimo a scopo di guadagno, sobbarcandosi le peggiori fatiche, e gli propone invece una vita di disimpegno: indulge gnio, carpamus dulcia, nostrum est / quod vivis, cinis et manes et fabula fieds; / vive memor leti, fugit hora, hoc quod loquor inde est (vv. 151-153). Si noti che l'invito a vivere ricordando sempre l'incombere della morte (vive memor lett) è certamente consono all'esortazione epicureizzante di Mollezza, ma potrebbe rivelare anche un tratto tipico del sentire etrusco, il pervasivo senso della morte, che da più parti è stato rilevato.

${ }_{21}$ At si unctus cesses et figas in cute solem, / est prope te ignotus, cubito qui tangat et acre / despuat: "Hi mores, penemque arcanaque lumbi / runcantem, populo marcentis pandere vulvas! / Tu cum maxillis balanatum gausape pectas, / inguinibus quare detonsus gurgulio exstat? / Quinque palaestritae licet haec plantaria vellant, / elixasque nates labefactent forcipe adunca, / non tamen ista filix ullo mansuescit aratro». 
vedremo, fu amico di Trasea. Come ci informa la Vita Persi, 4-5, alla scuola dello stoico L. Anneo Cornuto -liberto della famiglia degli Annei, esiliato poi da Nerone nel 66 d.C.- ${ }^{22}$ Persio conobbe il poeta stoico Lucano, che fu un suo estimatore, e anche Seneca, che tuttavia non ammirò in modo particolare ${ }^{23}$; soprattutto, Persio fu molto vicino, tanto da viaggiare più volte insieme con lui- a Trasea Peto (ibid.) ${ }^{24}$, il senatore stoico profondamente legato al mondo etrusco e imparentato con lo stesso Persio grazie alla moglie. Trasea, infatti, era marito di Arria Minore, cognata di Persio e figlia di Arria Maggiore, che fu la moglie eroica di Cecina Peto: il suo stoico suicidio al cospetto del marito, che ella invitò a prendere il pugnale con le celebri parole Paete, non dolet, fu tanto ammirato da Persio che egli vi dedicò dei versi: il significato politico di questi versi di opposizione era tale che Cornuto poi si adoperò, come accennavo, perché non venissero pubblicati (Vita, 8). La vicinanza politica di Persio a Trasea e la sua opposizione al principato neroniano è documentabile, del resto, anche attraverso ulteriori elementi.

È vero che Persio -il quale, come Musonio, era di famiglia non senatoria ma equestre, sia pur legata a membri del ceto senatorio (Vita, 1-2) - ${ }^{25}$, a differenza di Trasea, non fece attività di opposizione diretta o aperta contro il principato, non subì persecuzioni e morì, appena ven-

22 Su cui mi limito a rinviare a G.W. Most, Cornutus and Stoic Allegoresis. A Preliminary Report, in ANRW, II 36, 3, Berlin-New York 1989, pp. 2014-2065 e al mio cit. Anneo Cornuto, Compendio di teologia greca.

${ }_{23}$ Cognovit per Cornutum etiam Annaeum Lucanum, aeque tum auditorem Cornuti ... qui libros philosophiae reliquit. Sed Lucanus adeo mirabatur scripta Flacci, ut vix se retineret, recitante eo, a clamore: quae ille, esse vera poemata: quae ipse faceret, ludos. Sero cognovit et Senecam, sed non ut caperetur eius ingenio.

${ }_{24}$ Idem decem fere annis summe dilectus a Paeto Thrasea est, ita ut peregrinaretur quoque cum eo aliquando, cognatam eius Arriam uxorem habente.

${ }_{25}$ Natus in Etruria Volaterris, eques Romanus, sanguine et affinitate primi ordinis viris coniunctus. Sull'opposizione al principato in età neroniana: B. MAC ALIDON, Senatorial Opposition to Claudius and Nero, "American Journal of Philology", 77 (1956), pp. 113 ss.; B. BALDWIN, Executions, Trials and Punishments in the Reign of Nero, "La Parola del Passato" 22 (1967), págs 425 ss.; E. CIzEK, L'époque de Néron et ses controverses idéologiques, Leiden 1962; P. A. Brunt, Stoicism and the Principate, "Papers of the British School at Rome", 43 (1975), pp. 7 ss.; E. WIStRand, The Stoic Opposition to the Principate, «Studii Clasice» 18 (1979), pp. 93 ss.; soprattutto V. RuDich, Political Dissidence under Nero. The Price of Dissimulation, London - New York 1993. Per il rapporto di Persio con questa opposizione Trinvio a D. Bo, Persio e l'opposizione anti-neroniana, in Filologia e forme letterarie. Studi offerti a F. Della Corte, I-V, Urbino 1987, III, pp. 403-418.

${ }_{26}$ Ce ne informa la Vita Persi che in passato è stata attribuita a Svetonio: Persio morì di una malattia di stomaco, non ancora trentenne (Vita, 9), il 24 novembre del 62 (Vita, 1). 
tottenne, di morte naturale ${ }^{26}$. Tuttavia, la Vita Persi, 10 , ci parla di una critica, sia pure velata, del poeta nei confronti di Nerone ed è possibile trovare altri indizi, nella lettura delle satire persiane, di un atteggiamento polemico di questo tipo. La notizia della Vita è, come è noto, che Persio abbia inteso alludere a Nerone nel verso "auriculas asini Mida rex habet»: così infatti suonava originariamente I 121. Proprio per timore di una reazione di Nerone, appena dopo la morte di Persio (62 d.C.) Cornuto, maestro del poeta e suo caro amico, trovandosi a pubblicare le satire, preferi prudentemente mutare il verso in "auriculas asini quis non habet?". Evidentemente, la menzione del Mida rex poteva destare sospetti e indurre ad identificare il rex con l'imperatore in carica, senza contare che Nerone era accusato fin dai contemporanei di eccessiva avidità (Giovenale stesso condanna a più riprese la brama di ricchezze di Nerone ${ }^{27}$ ) e dunque era possibile che il mitico Mida servisse da metafora per indicare l'imperatore contemporaneo assetato d'oro. II biografo sostiene (Vita, 10) ${ }^{28}$ che fu la critica ai poeti e agli oratori contemporanei a portare Persio ad attaccare anche Nerone ${ }^{29}$.

27 $\times$ 15-18 Nerone è accusato di essersi voluto impossessare dei beni di Longino, di Seneca e dei Laterani ed i suoi sono detti dira tempora; ancora, a XII 128-30 Nerone è ricordato per le sue immense rapine: infatti a Pacuvio, che sarebbe disposto a sacrificare la figlia pur di ereditare, Giovenale augura di poter accumulare un patrimonio smisurato quanto i beni che Nerone rapinò, e di non amare alcuno, né essere amato da nessuno. L'amore per il lusso e la smodatezza di Nerone sono bersaglio di IV 136-143. Si veda per questo il mio L'opposizione allimpero in Giovenale, in L'opposizione nel mondo antico, a c. di M. Sord, Milano 2000, pp. 195-214.

28 Lecto Lucili libro decimo vehementer saturas componere instituit, cuius libri principium imitatus est, sibi primo, mox omnibus detrectaturus cum tanta recentium poetarum et oratorum insectatione ut etiam Neronem principem illius temporis inculpaverit. Cuius versus in Neronem cum ita se haberet: 'auriculas asini Mida rex habet', in eum modo a Cornuto, ipse tantummodo, est emendatus: 'auriculas asini quis non habet?', ne hoc in se Nero dictum arbitraretur. A commento di questo passo-chiave si vedano: J. P. Sullivan, Ass's Ears and Artises. Persius and Nero, "American Journal of Philology" 99 (1978), pp. 159-170; S. Koster, Der Baber des Mida oder Persius und Nero, in Ylle ego qui, Dichter zwischen Wort und Macht, Erlangen 1988, Erl. Forsch. R. A. 42.

${ }_{29}$ Nelle satire di Persio è poi possibile cogliere una feroce parodia di Caligola: Persio sta svolgendo un'argomentazione contro un eventuale erede che gli imponesse di risparmiare, per lasciargli un patrimonio maggiore alla morte: il poeta rivendica la libertà di gettare il proprio denaro nelle spese più futili e sceglie come esempio proprio l'occasione del finto trionfo di Caligola sui Germani (VI 43-47). E' celebre la parodistica descrizione del trionfo organizzato dalla moglie dell'imperatore, Cesonia, con attori imparruccati in luogo dei prigionieri ed altri simili dettagli coreografici ( $\mathrm{O}$ caro, forse non lo sai? Da Cesare è stata mandata una lettera incoronata d'alloro [missa est a Caesare laurus]/ per annunciare la grande strage [insignem ob cladem] della gioventù germanica, e dagli altari/ si scuote via la fredda cenere; e già Cesonia sta noleggiando armi da appendere alle porte/ e mantelli di re, e bionde parrucche per 
Ma Persio non critica soltanto singole figure di imperatori, per altro le più esecrate e colpite pubblicamente dalla damnatio memoriae. Egli è consapevole della profonda responsabilità che investe chiunque detenga il potere politico: un'intera satira è dedicata a questo tema. Alla sat. IV infatti si potrebbe dare il titolo: La fondazione etica dell'attività politica. Persio, ponendosi come un Socrate di fronte ad un giovane Alcibiade, obietta al suo interlocutore che egli non può svolgere l'attività politica (rem populi tractare, v. 1), poiché non sa distinguere il giusto dall'ingiusto, il bene dal male (IV 9-10) e non sa quale sia il sommo bene (IV 16). Per conseguenza, se il giovane politico è schiavo delle proprie passioni, come l'avidità e l'intemperanza, inutilmente si presenterà al popolo come virtuoso, facendosi passare per quel che non è. Questo è quindi il celebre ammonimento finale: «respingi quel che non sei [respue quod non es]: il popolino si riprenda i suoi regali:/ rientra nella tua dimora [tecum habita] e ti renderai conto di quanto siano scarse le tue suppellettili [quam sit tibi curta supellex]" (IV 51-52). Le suppellettili sono chiaramente le virtù: se mancano, è pecca ancor più grave cercare di simularle per detenere il potere politico. Sotto l'impero, però, la possibilità di essere politicamente davvero attivi era molto ridotta rispetto ai tempi della repubblica ed è innegabile che colui nelle cui mani era la summa del potere politico fosse appunto l'imperatore. Persio in questa satira sembra voler responsabilizzare proprio l'imperatore, avvisandolo che il suo potere deve essere fondato sulla sua rettitudine morale, e che la pratica della filosofia, intesa primariamente come etica -come la intendono in generale gli Stoici romani-, è imprescindibile per il sovrano ${ }^{30}$ : erano questi gli anni in cui Seneca appoggiava Nerone e lo guidava nel suo difficile ruolo; Persio, a causa della sua morte prematura, non vide la totale degenerazione del principato neroniano, però certamente ne vide le premesse. II tono della satira, rivolta ad un giovane che rem populi tractat, ma che è avido e intemperante e che simula virtù che non possiede, sembra quasi sug-

gli sconfitti, / carri da guerra e grandi statue del Reno"). II poeta prosegue fingendo di volere unirsi alle manifestazioni del trionfo ed ironizzando (VI 48-50): "agli dèi, dunque, e al genio del condottiero [genioque ducis] voglio allestire uno spettacolo/ con cento coppie di gladiatori, per queste gesta eccezionali [ob res egregie gestas] [...] voglio elargire al popolino olio, pane e carne".

30 Musonio Rufo, accanto a Seneca e a Persio, ha insistito in modo particolare sull'importanza della pratica filosofica da parte del sovrano: una delle Diatribe raccolte da Lucio è dedicata interamente a questo tema. 
gerire che il filosofo voglia istruire l'imperatore sulle responsabilità che il suo potere invece comporta. Qualora infatti il princeps non sia virtuoso, il rischio, gravissimo, è quello della degenerazione del principato in tirannide. Persio, in un passo della sat. III citato per la sua importanza anche da S. Agostino (Civ. Dei II 7), assegna alla somma divinità, Giove, il compito di vigilare sulla moralità di coloro che detengono il potere politico, pregandolo di intervenire contro i tiranni che si lasciano trascinare dalle loro passioni. La punizione invocata da Persio consiste nel mostrare loro la virtù, suscitando così in essi fieri rimorsi per averla abbandonata: «Magne pater divum, saevos punire tyrannos / haut alia ratione velis, cum dira libido / moverit ingenium ferventi tincta veneno: / virtutem videant intabescantque relicta" (III 35-38). Come per Seneca e per Musonio, anche per Persio, oppositore amico di Trasea Peto, il tema della tirannide è oggetto di seria riflessione. 